\title{
Antibacterial resistance in Helicobacter pylori strains isolated from Bulgarian children and adult patients over 9 years
}

\author{
Lyudmila Boyanova, ${ }^{1}$ Rossen Nikolov, ${ }^{2}$ Elena Lazarova, ${ }^{3}$ Galina Gergova, ${ }^{1}$ \\ Nikolai Katsarov, ${ }^{4}$ Victor Kamburov, ${ }^{5}$ Zoya Spassova, ${ }^{2}$ Sirigan Derejian, ${ }^{2}$ \\ Christo Jelev, ${ }^{3}$ Ivan Mitov ${ }^{1}$ and Zacharii Krastev ${ }^{2}$ \\ ${ }^{1}$ Department of Microbiology, Medical University of Sofia, Zdrave Street 2, 1431 Sofia, Bulgaria \\ ${ }^{2}$ Department of Gastroenterology, University Hospital St Ivan Rilski, Sofia, Bulgaria \\ ${ }^{3}$ Department of Gastroenterology, University Paediatric Hospital, Sofia, Bulgaria \\ ${ }^{4}$ Second Surgery Department of Alexander Hospital, Sofia, Bulgaria \\ ${ }^{5}$ Urgent Endoscopy Unit, Emergency Hospital Pirogov, Sofia, Bulgaria
}

\begin{abstract}
The aim of this study was to evaluate the primary, combined and post-treatment antibacterial resistance rates in 1205 Helicobacter pylori strains from non-treated (786 adults, 282 children) and treated (109 adults, 28 children) patients in Bulgaria. Susceptibility was tested by the limited agar dilution method. Overall primary resistance rates to metronidazole, clarithromycin, amoxicillin, tetracycline and both metronidazole and clarithromycin were respectively 15.0, 12.5, 1.5, 3.4 and $4 \cdot 7 \%$ in children and $25 \cdot 6,12 \cdot 6,0 \cdot 8,5 \cdot 2$ and $4.9 \%$ in adults. Primary metronidazole resistance in adults was more common than in children, but the differences for other agents tested were not significant. Primary resistance rates were in the range of those reported worldwide. There was no significant increase in primary resistance rates from 1996/1999 to $2003 / 2004$; however, clarithromycin resistance rates exhibited a slight tendency to increase. Post-treatment resistance to amoxicillin was detected in $1.6 \%$ of 63 strains. Post-treatment resistance to metronidazole was common ( $81.6 \%)$ and that to clarithromycin was considerable $(36 \%)$. Alarming emergence of strains with triple resistance to amoxicillin, metronidazole and clarithromycin was found in two non-treated and three treated patients. The results motivate a larger and continuing surveillance of $H$. pylori resistance in Bulgaria and worldwide.
\end{abstract}

Received 21 June 2005

Accepted 8 September 2005 previous or the national consumption of a given antibacterial agent, has been observed in some countries (Meyer et al., 2002; Megraud, 2004). The aim of this study was to assess the rates of the primary, combined, post-treatment and secondary antibacterial resistance in $\mathrm{H}$. pylori clinical isolates from 1205 patients with gastroduodenal diseases over 9 years in Bulgaria.

\section{METHODS}

Consecutive H. pylori strains isolated from non-treated patients (282 children and 786 adults) were evaluated in 1996-2004 and consecutive $H$. pylori strains from treated patients (109 adults and 28 children) were evaluated in 1998-2004. A single antral biopsy specimen was taken from the children and two antral biopsy specimens per patient were taken from the adults. Specimens from the treated patients were taken 4-8 weeks after the end of therapy for $H$. pylori eradication. Most common eradication regimens involved amoxicillin, clarithromycin and PPI (14 patients), amoxicillin, azithromycin and PPI (27), amoxicillin, metronidazole and PPI (26), amoxicillin, metronidazole and bismuth compound (6) and clarithromycin, 
metronidazole and PPI (5). No detailed data for the previous treatment of the other patients were available. The PPI used were omeprazole (the most common PPI used), rabeprazole and esomeprazole.

Stomach biopsy specimens were transported in Stuart transport medium (Merck) for $<5 \mathrm{~h}$. A smear was prepared from part of the specimen and was used for modified Gram staining with carbol fuchsin. Part of the specimen was placed in $10 \%$ urea agar medium and the colour change was read after incubation at $37^{\circ} \mathrm{C}$ for $30 \mathrm{~min}$ and $3 \mathrm{~h}$. The remaining part of the specimen was homogenized in sterile saline with sterile needles and inoculated onto blood agar (Columbia agar base; Becton Dickinson) with $10 \mu \mathrm{g}$ vancomycin, $5 \mu \mathrm{g}$ trimethoprim, $5 \mu \mathrm{g}$ cefsulodin and $5 \mu \mathrm{g}$ amphotericin $\mathrm{B} \mathrm{ml}^{-1}$ and/or $10 \%$ defibrinated sheep blood, and $1 \%$ IsovitaleX (Becton Dickinson). Both selective and non-selective media were used for primary culture. Plates were incubated microaerophilically for 12 days. Identification was made by Gram staining of the colonies, lack of aerobic growth on blood agar plates and testing for urease, oxidase and catalase.

Susceptibility was tested by a limited agar dilution method. Two drops (about $60 \mu \mathrm{l}$ ) of $\mathrm{H}$. pylori suspensions, prepared in Mueller-Hinton broth [National Centre of Infectious and Parasitic Diseases (NCIPD)] to obtain McFarland turbidity standard 3-4, were inoculated on Mueller-Hinton blood agar plates (NCIPD) containing $1 \%$ IsovitaleX and one of the following drug concentrations: 8,16 or $32 \mu \mathrm{g}$ metronidazole $\mathrm{ml}^{-1}, 0 \cdot 25,0 \cdot 5,1$ or $2 \mu \mathrm{g}$ clarithromycin $\mathrm{ml}^{-1}, 0 \cdot 5,1$ or $2 \mu \mathrm{g}$ amoxicillin $\mathrm{ml}^{-1}$ and $4 \mu \mathrm{g}$ tetracycline $\mathrm{ml}^{-1}$. Antimicrobial agents were obtained from Sigma (amoxicillin, metronidazole and tetracycline) and Abbott Laboratories (clarithromycin). The plates were incubated microaerophilically at $37^{\circ} \mathrm{C}$ for $2-3$ days. If $H$. pylori growth appeared on the plate, the isolate was considered as resistant to the corresponding drug concentration. Non-selective medium plates were used for a control of strain viability. The cut-off concentrations for resistance were: $>8 \mu \mathrm{g}$ metronidazole $\mathrm{ml}^{-1}$, $>1 \mu \mathrm{g}$ clarithromycin $\mathrm{ml}^{-1},>0.5 \mu \mathrm{g}$ amoxicillin $\mathrm{ml}^{-1}$ and $>4 \mu \mathrm{g}$ tetracycline $\mathrm{ml}^{-1}$ (Megraud et al., 1999; NCCLS, 2000). Primary resistance was defined as resistance of an $H$. pylori strain isolated from a patient with no previous therapy for $H$. pylori eradication. Post-treatment resistance was determined as resistance to a given antibacterial agent after therapy involving the agent, regardless of the strain sensitivity before treatment. Secondary resistance was defined as resistance acquired during treatment by a strain that was susceptible to the agent before the treatment. Secondary resistance to amoxicillin and macrolides was assessed in 18 and 13 strains, respectively.

Differences between patients with susceptible and resistant strains were assessed with a chi-squared test with or without Yates' correction. Yates' correction factor for continuity was added in the calculation of chi-squared values for $2 \times 2$ tables when the expected frequency was $<10$ in one or more cells.

\section{RESULTS}

Overall primary resistance rates to metronidazole, clarithromycin, amoxicillin, tetracycline and both metronidazole and clarithromycin were respectively $15 \cdot 0,12 \cdot 5,1 \cdot 5,3 \cdot 4$ and $4 \cdot 7 \%$ in children and $25 \cdot 6,12 \cdot 6,0 \cdot 8,5 \cdot 2$ and $4 \cdot 9 \%$ in adults. Primary metronidazole resistance in adults was more common than in children $(P<0 \cdot 001)$, but the differences between the age groups for the other antibacterial agents were not significant $(P>0 \cdot 20)$. There were no significant differences between primary resistance rates in adults aged $>60$ years and those aged $19-59$ years $(P>0 \cdot 20)$.

There was no significant increase in antimicrobial resistance rates in children and adults from 1996/1999 to 2003/2004 $(P>0 \cdot 20)$ (Table 1); however, the rates of clarithromycin resistance exhibited a slight tendency to increase in children (from $10 \cdot 4$ to $13.9 \% ; P>0 \cdot 20$ ) and in adults (from $9 \cdot 8$ to $14 \cdot 3 \% ; P>0 \cdot 20)$.

Table 1. Evolution of primary $H$. pylori resistance to antibacterial agents in Bulgaria

$\% \mathrm{R}$ is the percentage of resistant strains; $95 \% \mathrm{CI}$ is the $95 \%$ confidence interval for \% $\mathrm{R}$.

\begin{tabular}{|c|c|c|c|c|c|c|c|}
\hline \multirow[t]{2}{*}{ Agent } & \multirow[t]{2}{*}{ Years } & \multicolumn{3}{|c|}{ Children } & \multicolumn{3}{|c|}{ Adults } \\
\hline & & $\begin{array}{l}\text { Strains } \\
\quad(n)\end{array}$ & $\% \mathbf{R}$ & $95 \%$ CI & $\begin{array}{l}\text { Strains } \\
\quad(n)\end{array}$ & $\% \mathrm{R}$ & $95 \% \mathrm{CI}$ \\
\hline \multirow[t]{3}{*}{ Metronidazole } & 1996-1999 & 47 & $12 \cdot 8$ & $3 \cdot 1-22 \cdot 5$ & 120 & $27 \cdot 5$ & $19 \cdot 5-35 \cdot 5$ \\
\hline & 2000-2002 & 160 & $16 \cdot 2$ & $10 \cdot 5-21 \cdot 9$ & 286 & $28 \cdot 3$ & $23 \cdot 1-33 \cdot 5$ \\
\hline & 2003-2004 & 72 & $13 \cdot 9$ & $5 \cdot 9-21 \cdot 9$ & 374 & $23 \cdot 0$ & $18 \cdot 7-27 \cdot 3$ \\
\hline \multirow[t]{3}{*}{ Clarithromycin } & 1996-1999 & 48 & $10 \cdot 4$ & $1 \cdot 7-19 \cdot 1$ & 112 & $9 \cdot 8$ & $4 \cdot 3-15 \cdot 3$ \\
\hline & 2000-2002 & 161 & $12 \cdot 4$ & $7 \cdot 3-17 \cdot 5$ & 286 & $11 \cdot 5$ & $7 \cdot 8-15 \cdot 2$ \\
\hline & 2003-2004 & 72 & $13 \cdot 9$ & $5 \cdot 9-21 \cdot 9$ & 378 & $14 \cdot 3$ & $10 \cdot 8-17 \cdot 8$ \\
\hline \multirow[t]{3}{*}{ Amoxicillin } & 1996-1999 & 38 & $7 \cdot 9$ & $0-16 \cdot 6$ & 56 & 0 & $0-3 \cdot 3$ \\
\hline & 2000-2002 & 161 & 0 & $0-1 \cdot 2$ & 286 & $0 \cdot 3$ & $0-0 \cdot 9$ \\
\hline & 2003-2004 & 73 & $1 \cdot 4$ & $0-4 \cdot 1$ & 380 & $1 \cdot 3$ & $0 \cdot 2-2 \cdot 4$ \\
\hline \multirow[t]{3}{*}{ Tetracycline } & 1996-1999 & 38 & $2 \cdot 6$ & $0-7 \cdot 7$ & 70 & $4 \cdot 3$ & $0-9 \cdot 1$ \\
\hline & 2000-2002 & 135 & $3 \cdot 7$ & $0 \cdot 5-6 \cdot 9$ & 256 & $2 \cdot 3$ & $0 \cdot 5-4 \cdot 1$ \\
\hline & 2003-2004 & 64 & $3 \cdot 1$ & $0-7 \cdot 4$ & 366 & $7 \cdot 4$ & $4 \cdot 7-10 \cdot 1$ \\
\hline \multirow{3}{*}{$\begin{array}{c}\text { Metronidazole + } \\
\text { clarithromycin }\end{array}$} & 1996-1999 & 47 & $4 \cdot 2$ & $0-10 \cdot 0$ & 111 & $3 \cdot 6$ & $0 \cdot 1-7 \cdot 1$ \\
\hline & $2000-2002$ & 160 & $4 \cdot 4$ & $1 \cdot 2-7 \cdot 6$ & 286 & $4 \cdot 5$ & $2 \cdot 1-6 \cdot 9$ \\
\hline & 2003-2004 & 70 & $5 \cdot 7$ & $0 \cdot 2-11 \cdot 2$ & 372 & $5 \cdot 6$ & $3 \cdot 3-7 \cdot 9$ \\
\hline
\end{tabular}


The overall resistance rates to metronidazole, clarithromycin, amoxicillin, tetracycline and both metronidazole and clarithromycin in treated patients were $30 \cdot 4,18 \cdot 2,4 \cdot 3,0$ and $4 \cdot 5 \%$ in children and $59 \cdot 2,31 \cdot 3,4 \cdot 9,4 \cdot 3$ and $18 \cdot 5 \%$ in adults, respectively. Treated adult patients harboured fourfold more strains $(18 \cdot 5 \%)$ resistant to both metronidazole and clarithromycin than did the children $(4.5 \%)$. Posttreatment resistance rates were: amoxicillin, $1.6 \%$; metronidazole, $81 \cdot 6 \%$; and clarithromycin, $36.0 \%$ (Table 2). Post-treatment resistance was more common than primary resistance to metronidazole $(P<0 \cdot 001)$ and clarithromycin $(P<0 \cdot 001)$. Secondary resistance to clarithromycin was found in $3(23 \cdot 1 \%)$ of 13 cases. Secondary resistance to amoxicillin was not detected in 18 cases evaluated.

\section{DISCUSSION}

Primary resistance rates of $H$. pylori were in the range of those reported in other countries (Megraud, 2004), with relatively higher resistance rates to amoxicillin and tetracycline and a lower resistance rate to metronidazole in Bulgarian children. Primary metronidazole resistance was more common in adults than in children, probably because of the use of nitroimidazoles to treat gynaecological and dental infections in adults.

H. pylori resistance to amoxicillin and tetracycline has been reported only in some countries (Meyer et al., 2002; Megraud, 2004). The detection of two strains with triple resistance to amoxicillin, clarithromycin and metronidazole from a non-treated man (in 2003) and a boy aged 14 years (in 2004) is worrying. This focuses attention on possible multidrug resistance in some $H$. pylori strains and related future therapeutic problems. Combined $H$. pylori resistance to beta-lactams, nitroimidazoles, tetracycline and other agents has been reported recently (Kwon et al., 2003).

Primary $H$. pylori resistance rates to clarithromycin or metronidazole have exhibited a significant increase or only slight differences over time according to different authors (Lopez-Brea et al., 2001; Bontems et al., 2001; Kalach et al., 2001). In the present study, no significant increase in

Table 2. Post-treatment resistance in $H$. pylori

\begin{tabular}{|llccc|}
\hline Agent & Group & Strains $(\boldsymbol{n})$ & \% R & $\mathbf{9 5 \% ~ C I}$ \\
\hline Amoxicillin & Adults & 46 & $2 \cdot 2$ & $0-6 \cdot 5$ \\
& Children & 17 & 0 & $0-9 \cdot 8$ \\
& Total & 63 & $1 \cdot 6$ & $0-4 \cdot 7$ \\
Metronidazole & Adults & 30 & $86 \cdot 7$ & $74 \cdot 3-99 \cdot 1$ \\
& Children & 8 & $62 \cdot 5$ & $26 \cdot 6-98 \cdot 4$ \\
& Total & 38 & $81 \cdot 6$ & $69 \cdot 1-94 \cdot 1$ \\
Clarithromycin & Adults & 39 & $41 \cdot 0$ & $25 \cdot 4-56 \cdot 6$ \\
& Children & 11 & $18 \cdot 2$ & $0-42 \cdot 1$ \\
& Total & 50 & $36 \cdot 0$ & $22 \cdot 6-49 \cdot 4$ \\
\hline
\end{tabular}

primary antibacterial resistance rates was detected in children and adults from 1996/1999 to 2003/2004; however, the clarithromycin resistance rate exhibited a slight tendency to increase. The results could reflect the relatively low macrolide consumption in Bulgaria [0.34 defined daily doses per 1000 inhabitants per day (DID) in 1999], the moderate nitroimidazole consumption (0.41 DID in 1999) and the rare use of metronidazole in children. The primary tetracycline resistance rate (low but constant since 1999) can be associated with the still frequent tetracycline consumption in our country (4.24 DID in 1999). Amoxicillin resistance was rare but was detected in 1999, 2001, 2003 and 2004.

Amoxicillin resistance (MIC 1-2 $\mu \mathrm{g} \mathrm{ml}^{-1}$ ), combined with metronidazole resistance, was found in four strains from treated patients. Three of the strains also exhibited clarithromycin resistance. Amoxicillin resistance has been associated with a mutation in the $p b p-1 A$ gene and altered uptake of beta-lactams after long exposure of $H$. pylori to amoxicillin (DeLoney \& Schiller, 2000). Japanese authors have reported an increase in $H$. pylori resistance rates to amoxicillin from 2000 to 2003 (Watanabe et al., 2005).

In conclusion, the primary resistance rates of $H$. pylori from Bulgarian patients were in the range of those reported worldwide. There was no significant increase in resistance rates to antimicrobial agents in children and adults from $1996 / 1999$ to $2003 / 2004$, although the rates of clarithromycin resistance exhibited a slight tendency to increase. Since 1999, tetracycline-resistant strains have been detected every year, and amoxicillin resistance has been observed less frequently. The alarming appearance of strains with triple resistance to amoxicillin, metronidazole and clarithromycin was detected in non-treated and treated patients. The posttreatment resistance rate in $H$. pylori to metronidazole was high and that to clarithromycin was considerable. The present results motivate the need for a larger and continuing surveillance of $H$. pylori resistance in our country and worldwide.

\section{REFERENCES}

Bontems, P., Devaster, J. M., Corvaglia, L., Dezsofi, A., Van Den Borre, C., Goutier, S., Butzler, J. P. \& Cadranel, S. (2001). Twelve year observation of primary and secondary antibiotic-resistant Helicobacter pylori strains in children. Pediatr Infect Dis J 20, 1033-1038.

Cantón, R., Martín de Argila, C., de Rafael, L. \& Baquero, F. (2001). Antimicrobial resistance in Helicobacter pylori. Rev Med Microbiol 12, 47-61.

DeLoney, C. R. \& Schiller, N. L. (2000). Characterization of an in vitro-selected amoxicillin-resistant strain of Helicobacter pylori. Antimicrob Agents Chemother 44, 3368-3373.

Kalach, N., Bergeret, M., Benhamou, P. H., Dupont, C. \& Raymond, J. (2001). High levels of resistance to metronidazole and clarithromycin in Helicobacter pylori strains in children. J Clin Microbiol 39, 394-397.

Kwon, D. H., Dore, M. P., Kim, J. J., Kato, M., Lee, M., Wu, J. Y. \& Graham, D. Y. (2003). High-level $\beta$-lactam resistance associated with 
acquired multidrug resistance in Helicobacter pylori. Antimicrob Agents Chemother 47, 2169-2178.

Lopez-Brea, M., Martinez, M. J., Domingo, D. \& Alarcon, T. (2001). A 9 year study of clarithromycin and metronidazole resistance in Helicobacter pylori from Spanish children. J Antimicrob Chemother 48, 295-297.

Megraud, F. (2004). H. pylori antibiotic resistance: prevalence, importance, and advances in testing. Gut 53, 1374-1384.

Megraud, F., Lehn, N., Lind, T. \& 7 other authors (1999). Antimicrobial susceptibility testing of Helicobacter pylori in a large multicenter trial: the MACH 2 study. Antimicrob Agents Chemother 43, 2747-2752.

Meyer, J. M., Silliman, N. P., Wang, W. \& 7 other authors (2002). Risk factors for Helicobacter pylori resistance in the United States: the surveillance of $H$. pylori antimicrobial resistance partnership (SHARP) study, 1993-1999. Ann Intern Med 136, 13-24.

NCCLS (2000). Performance standards for antimicrobial susceptibility testing. Tenth informational supplement. Approved Standard M100S10 (M7). Wayne, PA: NCCLS.

Peitz, U., Sulliga, M., Wolle, K. \& 7 other authors (2002). High rate of post-therapeutic resistance after failure of macrolide-nitroimidazole triple therapy to cure Helicobacter pylori infection: impact of two second-line therapies in a randomized study. Aliment Pharmacol Ther 16, 315-324.

Watanabe, K., Tanaka, A., Imase, K., Tokunaga, K., Sugano, H., Kai, A., Ishida, H., Itoh, T. \& Takahashi, S. (2005). Amoxicillin resistance in Helicobacter pylori: studies from Tokyo, Japan from 1985 to 2003. Helicobacter 10, 4-11. 